\title{
HOMEOMORPHISMS OF BOUNDED LENGTH DISTORTION
}

\author{
JUSSI VÄISÄLÄ
}

\section{Introduction}

1.1. Let $D$ and $D^{\prime}$ be domains in the plane $R^{2}$, and let $f: D \rightarrow D^{\prime}$ be a homeomorphism. We let $l(\alpha)$ denote the length of a path $\alpha$. If $L \geqq 1$ and if

$$
l(\alpha) / L \leqq l(f \alpha) \leqq L l(\alpha)
$$

for all paths $\alpha$ in $D$, we say that $f$ is of $L$-bounded length distortion, abbreviated $L$-BLD. In a joint article [MV] of $O$. Martio and the author, we consider more general BLD maps: discrete open maps of domains of $R^{n}$ into $R^{n}$ satisfying (1.2). For homeomorphisms and, more generally, for immersions, (1.2) is equivalent to the following condition: Every point in $D$ has a neighborhood $U$ such that $f \mid U$ is $L$-bilipschitz, that is,

$$
|x-y| / L \leqq|f(x)-f(y)| \leqq L|x-y|
$$

for all $x, y \in U$. For this reason, the BLD immersions are often called locally bilipschitz maps or local quasi-isometries or just quasi-isometries [Jo], [Ge].

The BLD property can also be defined in terms of upper and lower derivatives. Let $L_{f}(x)$ and $l_{f}(x)$ be the upper and lower limits of $|f(x+h)-f(x)| /|h|$ as $h \rightarrow 0$. Then a homeomorphism $f$ is $L$-BLD if and only if $l_{f}(x) \geqq 1 / L$ and $L_{f}(x) \leqq L$ for all $x \in D$. In particular, if $f$ is differentiable at $x$, this means

$$
|h| / L \leqq\left|f^{\prime}(x) h\right| \leqq L|h|
$$

for all $h \in R^{2}$.

Every $L$-BLD homeomorphism is $L^{2}$-quasiconformal, but a quasiconformal map is BLD only if its derivative is a.e. bounded away from 0 and $\infty$.

The purpose of this paper is to identify the domains $D \subset R^{2}$ which are BLD homeomorphic to a disk or to a half plane. The corresponding problem for bilipschitz maps was solved in the early eighties by Tukia $\left[\mathrm{Tu}_{1}\right],\left[\mathrm{Tu}_{2}\right]$, Jerison-Kenig [JK] and Latfullin [La]; see also [Ge]. Their results can be stated as follows: A bounded domain $D$ is bilipschitz homeomorphic to a disk if and only if its boundary $\partial D$ is a rectifiable Jordan curve satisfying the chord-arc condition: There is $c \geqq 1$ such that

$$
\sigma(x, y) \leqq c|x-y|
$$


for all $x, y \in \partial D$; here $\sigma(x, y)$ is the length of the shorter arc of $\partial D$ between $x$ and $y$. The half plane case is similar; then $\partial D$ is a locally rectifiable Jordan curve through $\infty$ satisfying (1.5).

We show that the BLD homeomorphic images of the disk and the half plane can be characterized by a somewhat similar condition. However, the euclidean distance $|x-y|$ in (1.5) must be replaced by the internal distance $\lambda_{D}(x, y)$, which is the infimum of the lengths of all arcs joining $x$ and $y$ in $D$. Moreover, $\partial D$ need not be a Jordan curve. Hence we shall replace $\partial D$ by the prime end boundary $\partial^{*} D$. Alternatively, the condition can be expressed in terms of the neighborhood system of $\partial D$ in $D$. An equivalent condition has been considered by Pommerenke $\left[\mathrm{Po}_{2}\right]$.

In a forthcoming paper I shall apply the results of the present paper to show that a bounded domain is BLD homeomorphic to a disk if and only if $D \times R^{1}$ is quasiconformally equivalent to a ball.

1.6. Notation. If $x \in R^{n}$ and $r>0, B(x, r)$ is the open ball with center $x$ and radius $r$, and $S(x, r)$ is its boundary sphere. We shall write

$$
B(r)=B(0, r), \quad B^{n}=B(1), \quad S(r)=S(0, r), \quad S^{n-1}=S(1) .
$$

We let $d(A)$ denote the euclidean diameter of a set $A \subset R^{n}$, and $d(x, A)$ is the distance between $A$ and a point $x \in R^{n}$.

\section{Preliminaries}

In this section we introduce the internal chord-arc condition for simply connected domains in $R^{2}$. In 2.9 we give a modulus estimate needed in Section 3. Since it may have independent interest, it is formulated for an arbitrary dimension $n$.

2.1. Jordan domains. A domain $D \subset R^{2}$ is a Jordan domain if its boundary $\partial D$ in the extended plane $\dot{R}^{2}=R^{2} \cup\{\infty\}$ is a Jordan curve (homeomorphic to a circle). Suppose that $D$ is a Jordan domain and that $\partial D$ is locally rectifiable, that is, every compact arc in $\partial D \backslash\{\infty\}$ is rectifiable. If $a, b \in \partial D \backslash\{\infty\}$, we let $\sigma_{D}(a, b)$ denote the length of the shorter subarc of $\partial D$ with end points $a$ and $b$. If $x, y \in \bar{D} \backslash\{\infty\}$, $\lambda_{D}(x, y)$ will denote the infimum of the lengths of all paths joining $x$ and $y$ in $D$. We say that $D$ has the internal chord-arc property with parameter $c \geqq 1$ if

$$
\sigma_{D}(a, b) \leqq c \lambda_{D}(a, b)
$$

for all finite boundary points $a, b$ of $D$. We abbreviate this by saying that $D$ is $c$-ICA.

The ordinary chord-arc condition (1.5) clearly implies (2.2). One can show that a Jordan curve through $\infty$ satisfies (1.5) if and only if both components of its complement have the property (2.2). The domain $D=\left\{(x, y) \in R^{2}: x<0\right.$ or $\left.y>|x|^{2}\right\}$ satisfies (2.2) but not (1.5). 
2.3. Prime ends. We give a brief summary on some known facts on prime ends. In what follows, we assume that $D$ is a simply connected proper subdomain of $R^{2}$ which is finitely connected on the boundary. This means that every boundary point of $D$ has arbitrarily small neighborhoods $U$ such that $D \cap U$ has only a finite number of components. Equivalently, $\partial D$ is locally connected. Still equivalently, every QC map $f: B^{2} \rightarrow D$ has a continuous extension $\bar{f}: \bar{B}^{2} \rightarrow \bar{D}$. See [Nä, 3.2] and $\left[\mathrm{Po}_{1}, 9.8\right]$.

The prime ends of such a domain $D$ are always of the first kind and can be defined as equivalence classes of tails. By a tail of $D$ we mean a path $\alpha:[a, b) \rightarrow D$ such that $\alpha(t) \rightarrow z \in \partial D$ as $t \rightarrow b$. The point $z$ is written as $h(\alpha)$. A subtail of $\alpha$ is a restriction to a subinterval $\left[a_{1}, b\right)$. If $U$ is a neighborhood of $h(\alpha)$, there is a unique component $W(U, \alpha)$ of $U \cap D$ containing a subtail of $\alpha$. Two tails $\alpha$ and $\beta$ are equivalent if $h(\alpha)=h(\beta)$ and if $W(U, \alpha)=W(U, \beta)$ for every neighborhood $U$ of $h(\alpha)$. The equivalence class $\bar{\alpha}$ of a tail $\alpha$ is a boundary element of $D$, and their collection $\partial^{*} D$ is the prime end boundary of $D$. The set $D^{*}=D \cup \partial^{*} D$ has a natural topology such that $\left(D^{*}, \partial^{*} D\right)$ is homeomorphic to $\left(\bar{B}^{2}, S^{1}\right)$. In fact, every QC homeomorphism $f: B^{2} \rightarrow D$ has a unique extension to a homeomorphism $f^{*}: \bar{B}^{2} \rightarrow D^{*}$. There is a natural continuous impression map $i: D^{*} \rightarrow \bar{D}$, defined by $i(\bar{\alpha})=h(\alpha)$ for $\bar{\alpha} \in \partial^{*} D$ and by $i \mid D=$ id. If $D$ is locally connected at a boundary point $z, i^{-1}(z)$ consists of a single point, which is often identified with $z$. In particular, if $D$ is a Jordan domain, we can identify $\partial^{*} D=\partial D$ and $D^{*}=\bar{D}$.

Suppose that $\alpha$ is a subarc of $\partial^{*} D$.Then $i \mid \alpha$ is a path in $\dot{R}^{2}$ and has a well-defined length $l(\alpha) \in(0, \infty]$, called the length of $\alpha$. If $i(u)=\infty$ for at most one $u \in \partial^{*} D$, then also written as $\infty$, and if $l(\alpha)<\infty$ for every compact arc $\alpha \subset \partial^{*} D \backslash\{\infty\}$, we say that $\partial^{*} D$ is locally rectifiable. Equivalently, $l(\alpha)$ can be defined as the infimum of all numbers $\lambda$ such that there is a sequence of arcs $\alpha_{j} \subset D$ such that (1) $\alpha_{j} \rightarrow \alpha$ in the natural topology of the space of all arcs of $D^{*}$ and (2) $l\left(\alpha_{j}\right) \rightarrow \lambda$.

2.4. The ICA property. Suppose that $D$ is as in 2.3 and that $\partial^{*} D$ is locally rectifiable. If $u$ and $v$ are finite points in $\partial^{*} D$, we let $\sigma_{D}(u, v)$ denote the length of the shorter arc of $\partial^{*} D$ between $u$ and $v$. Furthermore, let $\lambda_{D}(u, v)$ be the infimum of the lengths of all paths $\alpha$ joining $u$ and $v$ in $D$. By this we mean that $\alpha$ is an open path which has subpaths representing both $u$ and $v$. One has always $\lambda_{D}(u, v) \leqq \sigma_{D}(u, v)$. If there is a constant $c \geqq 1$ such that

$$
\sigma_{D}(u, v) \leqq c \lambda_{D}(u, v)
$$

for all finite $u, v \in \partial^{*} D$, we say that $D$ is $c$-ICA.

For Jordan domains $D$, this definition is equivalent to that given in 2.1. The complement of a ray and a disk with a radial slit are ICA non-Jordan domains.

2.6. Remarks. 1. Pommerenke [ $\left[\mathrm{Po}_{2}\right.$, Theorem 2] considered domains $D$ satisfying the condition

$$
\sigma_{D}(u, v) \leqq c \delta_{D}(u, v)
$$


where $\delta_{D}(u, v)$ is the infimum of the diameters $d(|\alpha|)$ of all paths $\alpha$ joining $u$ and $v$ in $D$. Since $\delta_{D} \leqq \lambda_{D}$, (2.7) implies (2.5). Conversely, (2.5) implies that (2.7) is true with $c$ replaced by a constant $c_{1}=c_{1}(c)$. This follows easily from the results in Section 3 . The half plane with an orthogonal slit is $2^{1 / 2}$-ICA, but satisfies $(2.7)$ only for $c \geqq 2$.

2. It is possible to characterize the ICA property without mentioning prime ends: Let $D \subset R^{2}$ be simply connected, $D \neq R^{2}$. Then $D$ is $c$-ICA if and only if for each pair $a, b \in \partial D \backslash\{\infty\}$ and for each $\varepsilon>0$ there is $r>0$ such that if a path $\alpha$ joins points $x \in D \cap B(a, r)$ and $y \in D \cap B(b, r)$ in $D$, there is a path $\gamma$ joining $x$ and $y$ in $D \cap\left(\partial D+\varepsilon B^{2}\right)$ with $l(\gamma) \leqq c l(\alpha)+\varepsilon$.

3. One can also show that $D$ is $c$-ICA if and only if the chord-arc condition

$$
\sigma_{D}(u, v) \leqq c|i(u)-i(v)|
$$

is valid for all $u, v \in \partial^{*} D$ which are the end points of a segmental crosscut of $D$, that is, the open line segment with end points $i(u), i(v)$ lies in $D$ and represents both $u$ and $v$.

2.8. Path families. In Section 3 we shall consider paths $\gamma$ joining a boundary point $a \in \partial D$ to a point $b \in \bar{D}$ in $D$. Such a path defines an element $u \in \partial^{*} D$ with $i(u)=a$, and we can as well consider $\gamma$ as a path joining $u$ to $b$. If $\Gamma$ is a family of such paths, the modulus $M(\Gamma)$ is always well defined. If $\gamma$ is a path, we let $|\gamma|$ denote its locus im $\gamma$.

2.9. Lemma. Let $t>0$ and let $A \subset R^{n}$ with $d(A) \leqq t$. Let $\lambda>0$ and let $\Gamma$ be a family of paths in $R^{n}$ such that $l(\gamma) \geqq \lambda t$ and $\overline{|\gamma|} \cap A \neq \emptyset$ for all $\gamma \in \Gamma$. Then $M(\Gamma) \leqq \mu_{n}(\lambda)$, where $\mu_{n}(\lambda) \rightarrow 0$ as $\lambda \rightarrow \infty$.

Proof. We may assume that $t=1$ and that $A \subset \bar{B}^{n}$. We may also assume that $\lambda>1$, since otherwise $\left[\mathrm{V} \ddot{a}_{1}, 7.1\right]$ gives $M(\Gamma) \leqq m(B(2)) / \lambda^{n}$. Define $\varrho_{1}, \varrho_{2}: R^{n} \rightarrow R^{1}$ by

$$
\varrho_{1}(x)=\frac{2}{(\ln \lambda)|x|} \text { for } 1<|x|<\lambda^{1 / 2}, \quad \varrho_{2}(x)=1 / \lambda \text { for }|x|<\lambda^{1 / 2},
$$

and $\varrho_{j}(x)=0$ for other $x \in R^{n}$. We show that $\varrho=\max \left(\varrho_{1}, \varrho_{2}\right)$ belongs to $F(\Gamma)$, that is, the line integral of $\varrho$ along any rectifiable $\gamma \in \Gamma$ is at least one.

If $|\gamma| \subset B\left(\lambda^{1 / 2}\right)$, we have

$$
\int_{\gamma} \varrho_{2} d s \geqq l(\gamma) / \lambda \geqq 1
$$

If $|\gamma| \leftarrow B\left(\lambda^{1 / 2}\right), \overline{|\gamma|}$ meets the spheres $S^{n-1}$ and $S\left(\lambda^{1 / 2}\right)$, and hence

$$
\int_{\gamma} \varrho_{1} d s \equiv \int_{1}^{\lambda^{1 / 2}} \frac{2 d r}{r \ln \lambda}=1
$$


Thus $\varrho \in F(\Gamma)$, which implies

$$
M(\Gamma) \leqq \int_{R^{n}} \varrho^{n} d m
$$

Letting $\Omega$ and $\omega$ denote the volume of $B^{n}$ and the area of $S^{n-1}$ we have

$$
\int_{R^{n}} \varrho_{1}^{n} d m \leqq 2^{n-1} \omega(\ln \lambda)^{1-n}, \quad \int_{R^{n}} \varrho_{2}^{n} d m=\Omega \lambda^{-n / 2},
$$

and the lemma follows.

\section{Main results}

3.1. In this section we characterize the BLD homeomorphic images of $B^{2}$ and $H^{2}$. The half plane case is given in 3.4 and the disk case in 3.8. We recall from 1.1 that a homeomorphism $f: D \rightarrow D^{\prime}$ is $L$-BLD if

$$
l(\alpha) / L \leqq l(f \alpha) \leqq L l(\alpha)
$$

for every path in $D$ or, equivalently, $f$ is locally $L$-bilipschitz.

3.3. Theorem. Let $D \subset R^{2}$ be a convex Jordan domain, and let $f: D \rightarrow D^{\prime} \subset R^{2}$ be an L-BLD homeomorphism. Then:

(1) $f$ is L-Lipschitz in the euclidean metric.

(2) $D^{\prime}$ is finitely connected on the boundary.

(3) $f$ is L-bilipschitz in the metric $\lambda_{D^{\prime}}$.

(4) $\partial^{*} D^{\prime}$ is locally rectifiable.

(5) $f$ has a unique extension to a homeomorphism $f^{*}: \bar{D} \rightarrow D^{\prime *}$, which is $L$-bilipschitz outside $\infty$ in the metric $\lambda_{D^{\prime}}$.

(6) $f^{*} \mid \partial D$ is L-bilipschitz outside $\infty$ in the metrics $\sigma_{D}$ and $\sigma_{D^{\prime}}$.

If, in addition, $D$ has the c-chord-arc property, $D^{\prime}$ is $L^{2} c$-ICA.

Proof: Observe that since $D$ is convex, $\lambda_{D}$ is the euclidean metric. The condition (1) follows at once from convexity. Hence $f$ has a continuous extension $\bar{f}: \bar{D} \rightarrow \bar{D}^{\prime}$. Then (2) follows from [Nä, 3.2]. Since $D$ is convex, $\partial D$ is locally rectifiable. The rest of the theorem follows easily from (3.2) and from the considerations in 2.3 and 2.4.

3.4. Theorem. A simply connected domain $D \subset R^{2}$ is BLD homeomorphic to the half plane $H^{2}=\left\{(x, y) \in R^{2}: y>0\right\}$ if and only if (1) $D \neq R^{2}$, (2) $D$ is finitely connected on the boundary, (3) D is ICA, and (4) D is unbounded.

Proof. Suppose that $f: H^{2} \rightarrow D$ is an $L$-BLD homeomorphism. Since the image of the segment $\{0\} \times(0,1]$ has length at most $L,(1)$ is true. Since $H^{2}$ is convex and 1-ICA, (2) and (3) follow from 3.3. Since $f^{-1}$ is locally $L$-Lipschitz, $\infty=m\left(H^{2}\right) \equiv$ $L^{2} m(D)$, which implies (4). 
The converse part is considerably harder. We first give an outline of the proof. Suppose that $D$ satisfies the conditions (1)-(4). Choose a conformal map $f_{1}$ : $H^{2} \rightarrow D$. It has a homeomorphic extension, still written as $f_{1}: \bar{H}^{2} \rightarrow D^{*}$. We may assume that $f_{1}(\infty)=\infty$. Choose a homeomorphism $g: R^{1} \rightarrow \partial^{*} D \backslash\{\infty\}$ such that $\sigma_{D}(g(x), g(y))=|x-y|$ for all $x, y \in R^{1}$ and such that the homeomorphism $s: R^{1} \rightarrow R^{1}$ defined by $s(x)=g^{-1}\left(f_{1}(x)\right)$ is increasing. Extend $s$ by the Beurling-Ahlfors construction [Ah, p. 69] to a homeomorphism $f_{2}: \bar{H}^{2} \rightarrow \bar{H}^{2}$. Then $f=f_{1} f_{2}^{-1}: \bar{H}^{2} \rightarrow D^{*}$ is a homeomorphism, and $f \mid H^{2}$ will be the desired BLD homeomorphism.

Step 1. We show that $s$ is quasisymmetric (QS). Let $x \in R^{1}$ and $t>0$. Let $\Gamma$ be the family of all paths joining the intervals $[x-t, x]$ and $[x+t, \infty]$ in $H^{2}$. Then $M(\Gamma)=1$. If $\gamma$ belongs to the image $\Gamma^{\prime}$ of $\Gamma$ under $f_{1}, \gamma$ has end points $a, b$ with $a \in A=f_{1}[x-t, x]$ and $b \in B=f_{1}[x+t, \infty]$. The $\sigma$-diameter of $A$ is at most its length $s(x)-s(x-t)$, and hence $d(i A) \leqq s(x)-s(x-t)$. Furthermore, $\sigma_{D}(a, b) \geqq s(x+t)-$ $s(x)$. Since $D$ is $c$-ICA, this implies $s(x+t)-s(x) \leqq c l(\gamma)$. From 2.8 we obtain the estimate $M\left(\Gamma^{\prime}\right) \leqq \mu_{2}(R)$ with

$$
R c=\frac{s(x+t)-s(x)}{s(x)-s(x-t)} .
$$

Since $f$ is conformal, $M(\Gamma)=M\left(\Gamma^{\prime}\right)=1$. Since $\mu_{2}(R) \rightarrow 0$ as $R \rightarrow \infty$, we obtain an upper bound for $R c$. A lower bound is found similarly, changing the roles of $x-t$ and $x+t$. Hence $s$ is $H-\mathrm{QS}$ with a constant $H$ depending only on $c$.

Let $f_{2}: \bar{H}^{2} \rightarrow \bar{H}^{2}$ be the Beurling-Ahlfors extension of $s$. Then $f_{2} \mid H^{2}$ is $K$-QC and $L$-bilipschitz in the hyperbolic metric of $H^{2}$ [Ah, p. 73] with $L=L(c)$ and $K=L^{2}$. Then $f=f_{1} f_{2}^{-1}: \bar{H}^{2} \rightarrow D^{*}$ is a homeomorphism, and $f \mid R^{2}=g$; thus

$$
\sigma_{D}(f(x), f(y))=|x-y|
$$

for all $x, y \in R^{1}$.

Step 2. We write $\delta(w)=d(w, \partial D)$ for $w \in D$ and show that there is a constant $M=M(c)$ such that

$$
y / M \leqq \delta(f(z)) \leqq M y
$$

for every $z=(x, y) \in H^{2}$.

Let $T$ be the line through $b=i(f(x))$ and $f(z)$, let $R$ be the component of $T \backslash\{f(z)\}$ not containing $b$, and let $C^{\prime}$ be the component of $R \cap D$ with end point $f(z)$. Let $\Gamma$ be the family of all paths joining the real segment $[x, x+y]$ to $C=f^{-1} C^{\prime}$ in $H^{2}$. Then well known modulus estimates show that $M(\Gamma) \geqq q_{0}>0$ with a universal constant $q_{0}>0$, cf. [GV, Lemma 3.3, p. 13]. Assume that $\delta(f(z))=\delta>y$. Since (3.5) implies $\sigma_{D}(f(x+y), f(x))=y$, the members of $\Gamma^{\prime}=f \Gamma$ meet the circles $S(b, y)$ and $S(b, \delta)$. Hence $M\left(\Gamma^{\prime}\right) \leqq 2 \pi / \ln (\delta / y)$. Since $M(\Gamma) \leqq K M\left(\Gamma^{\prime}\right)$, we obtain the second inequality of (3.6) with $M=e^{2 \pi K / q_{0}}$.

We turn to the first inequality of (3.6). Fix $z=(x, y) \in H^{2}$ and set $\delta=\delta(f(z))$. Choose $w_{0} \in \partial D$ with $\left|w_{0}-f(z)\right|=\delta$. The segment $\left[f(z), w_{0}\right)$ defines an element 
$u_{0} \in \partial^{*} D$ with $i\left(u_{0}\right)=w_{0}$. Let $C_{0}^{\prime}$ be the arc on $\partial^{*} D$ such that $u_{0}$ divides $C_{0}^{\prime}$ to two subarcs of length $7 c \delta$. Let $C_{1}$ be the vertical ray with end point $z$. Then $C_{1}^{\prime}=f C_{1}$ joins $f(z)$ to $\infty$ in $D$. Let $J$ be the subarc of $C_{1}^{\prime}$ joining $f(z)$ and a point $w_{1} \in S(f(z), \delta)$ in $B(f(z), \delta)$.

Case 1. $\left|w_{1}-w_{0}\right| \geqq \delta$. Set $w_{2}=\left(w_{0}+f(z)\right) / 2$. For every $r \in\left[\delta / 2,3^{1 / 2} \delta / 2\right]$ we can choose an arc $\alpha_{r}$ of $S\left(w_{2}, r\right)$ with end points $a_{r} \in J, b_{r} \in \partial D$ and with $\alpha_{r} \backslash\left\{b_{r}\right\} \subset D$. Let $\Gamma^{\prime}$ be the family of all these arcs $\alpha_{r}$. A standard estimate gives $M(\Gamma) \geqq(\ln 3) / 4 \pi=$ $q_{1}$. The arc $\alpha_{r}^{\prime}=\alpha_{r} \backslash\left\{b_{r}\right\}$ defines an element $u_{r} \in \partial^{*} D$ with $i\left(u_{r}\right)=b_{r}$. Moreover, $\alpha_{r}^{\prime} \cup\left[a_{r}, w_{0}\right)$ joins $u_{r}$ and $u_{0}$ in $D$. Since $D$ is $c$-ICA, we have

$$
\begin{aligned}
\sigma_{D}\left(u_{r}, u_{0}\right) \leqq & c\left(l\left(\alpha_{r}\right)+\left|a_{r}-w_{0}\right|\right) \leqq c\left(2 \pi r+\left|a_{r}-w_{2}\right|+\left|w_{2}-w_{0}\right|\right) \\
& \leqq 3^{1 / 2} c \delta\left(2 \pi+3^{1 / 2} / 2+1 / 2\right) / 2<7 c \delta .
\end{aligned}
$$

Hence $b_{r} \in C_{0}^{\prime}$. Consequently, the members of $\Gamma=f^{-1} \Gamma^{\prime}$ join $C_{0}=f^{-1} C_{0}^{\prime}=$ $[x-7 c \delta, x+7 c \delta]$ and $C_{1}$. Thus either $y \leqq 7 c \delta$ or $M(\Gamma) \leqq 2 \pi / \ln (y / 7 c \delta)$. Since $M\left(\Gamma^{\prime}\right) \leqq K M(\Gamma)$, we obtain

$$
y \leqq 7 c \delta e^{2 \pi K / q_{1}},
$$

which yields the first inequality of (3.6).

Case 2. $\left|w_{1}-w_{0}\right|=t<\delta$. We repeat the argument of Case 1 replacing $w_{2}$ by $w_{3}=\left(w_{0}+w_{1}\right) / 2$. Since $S\left(w_{3}, r\right)$ meets $J$ and $\partial D$ whenever $t / 2 \leqq r \leqq 3^{1 / 2} t / 2$, we obtain the same estimate as in Case 1.

Step 3. We prove that the homeomorphism $f \mid H^{2}: H^{2} \rightarrow D$ is BLD. Since $f=f_{1} f_{2}^{-1}$ where $f_{1} \mid H^{2}$ is conformal and $f_{2} \mid H^{2} L$-bilipschitz in the hyperbolic metric, the diffeomorphism $f \mid H^{2}$ is $L$-bilipschitz in the hyperbolic metrics of $H^{2}$ and $D$. Hence

$$
|h| / L y \leqq \varrho(f(z))\left|f^{\prime}(z) h\right| \leqq L|h| / y
$$

for all $z=(x, y) \in H^{2}$ and $h \in R^{2}$, where $\varrho$ is the density of the hyperbolic metric in $D$. It is well known that

$$
1 / 4 \delta(w) \leqq \varrho(w) \leqq 1 / \delta(w)
$$

for all $w \in D$. Together with (3.6), these inequalities show that $f$ is $L_{1}$-BLD with $L_{1}=4 L M=L_{1}(c)$.

3.7. Remark. The proof above shows that the quantitative version of 3.4 is also true: If $f: H^{2} \rightarrow D$ is an $L$-BLD homeomorphism, $D$ is $c$-ICA with $c=L^{2}$. If $D$ is $c$-ICA and unbounded, there is an $L$-BLD homeomorphism $f: H^{2} \rightarrow D$ with $L=L(c)$.

3.8. Theorem. A simply connected domain $D \subset R^{n}$ is BLD homeomorphic to the unit disk $B^{2}$ if and only if (1) $D$ is finitely connected on the boundary, (2) $D$ is ICA, and (3) $D$ is bounded. 
Proof. Suppose that $f: B^{2} \rightarrow D$ is a BLD homeomorphism. Then $f$ is $L$ Lipschitz and hence $d(D) \leqq 2 L$. The conditions (1) and (2) follow from 3.3. More precisely, since $B^{2}$ is $\pi$-ICA, $D$ is $\pi L^{2}$-ICA.

The converse part is proved by modifying the proof of 3.4. Suppose that $D$ satisfies (1) and (3) and that $D$ is $c$-ICA. Then $\partial^{*} D$ is rectifiable. We normalize the situation by assuming $l\left(\partial^{*} D\right)=2 \pi$. Then there is a lengthpreserving homeomorphism $g: S^{1} \rightarrow \partial^{*} D$. Let $f_{1}: B^{2} \rightarrow D$ be a conformal map. It has an extension to a homeomorphism, still written as $f_{1}: \bar{B}^{2} \rightarrow D^{*}$. Then $g^{-1} f \mid S^{1}=s$ is a self homeomorphism of $S^{1}$, and $l(s \alpha)=l\left(f_{1} \alpha\right)$ for every arc $\alpha \subset S^{1}$. We may assume that $s \mid N_{3}=\mathrm{id}$ where $N_{3}=\left\{1, e^{2 \pi i / 3}, e^{4 \pi i / 3}\right\}$.

Step 1 . We show that $f_{1} \mid S^{1}$ has the following quasisymmetry property: If $\alpha$ and $\beta$ are adjacent arcs of $S^{1}$ with $l(\alpha)=l(\beta)$, then

$$
l\left(f_{1} \beta\right) \leqq c_{1} l\left(f_{1} \alpha\right)
$$

with some constant $c_{1}=c_{1}(c)$.

Assume first that $l(\alpha) \leqq \pi / 3$. Then we may assume that $\alpha \cup \beta$ does not meet the $\operatorname{arc} A=\left\{e^{i \varphi}: 2 \pi / 3<\varphi<4 \pi / 3\right\}$. Let $a$ be the end point of $A$ which has the greater distance from $\alpha \cup \beta$. Using the terminology of [LV, I.3.2] we consider the quadrilateral $Q$ consisting of the domain $B^{2}$, the three end points of $\alpha$ and $\beta$, and the point $a$. There are two path families $\Gamma_{1}, \Gamma_{2}$ associated with $Q$ with moduli $M\left(\Gamma_{1}\right)=1 / M\left(\Gamma_{2}\right)$. The length of a path in either family is at least $d(\alpha)=t$. Hence 2.9 implies $M\left(\Gamma_{j}\right) \leqq$ $\mu_{2}(1)$ and thus $M\left(\Gamma_{j}\right) \geqq 1 / \mu_{2}(1)$. Let $\Gamma_{1}$ be the family joining $\alpha$ to the opposite side of $Q$, and suppose that $\gamma \in f_{1} \Gamma_{1}=\Gamma_{1}^{\prime}$. The end points of $\gamma$ divide $\partial^{*} D$ into two arcs. One of these contains $f_{1} \beta$ and the other $f_{1} A$. Since $s A=A$, we have $l\left(f_{1} A\right)=$ $2 \pi / 3$. Since $D$ is $c$-ICA, this implies $c l(\gamma) \geqq \min \left(l\left(f_{1} \beta\right), 2 \pi / 3\right)$. Since $d\left(i f_{1} \alpha\right) \leqq l\left(f_{1} \alpha\right)$, 2.9 gives $M\left(\Gamma_{1}^{\prime}\right) \leqq \mu_{2}(R)$ with

$$
R=\frac{\min \left(l\left(f_{1} \beta\right), 2 \pi / 3\right)}{c l\left(f_{1} \alpha\right)} ;
$$

Since $M\left(\Gamma_{1}^{\prime}\right)=M\left(\Gamma_{1}\right) \geqq 1 / \mu_{2}(1)$ and since $\mu_{2}(t) \rightarrow 0$ as $t \rightarrow \infty, R$ is bounded by a universal constant $c_{0}$. Hence either (3.9) holds with $c_{1}=c_{0} c$ or $2 \pi / 3 \leqq c_{0} c l\left(f_{1} \alpha\right)$. In the latter case (3.9) holds with $c_{1}=2 c_{0} c$.

The case $l(\alpha)>\pi / 3$ reduces to the case above by dividing $\alpha$ and $\beta$ to three subarcs, cf. [LV, II.7.1].

Step 2. We want to extend $s: S^{1} \rightarrow S^{1}$ to a QC homeomorphism $f_{2}: \bar{B}^{2} \rightarrow \bar{B}^{2}$. To this end we choose an auxiliary Möbius map $h$ with $h B^{2}=H^{2}$ and $h(1)=\infty$. Then $s_{1}=h s h^{-1} \mid R^{1}$ is an increasing homeomorphism onto $R^{1}$. Moreover, $s_{1}$ is (weakly) $H$-QS with $H=H(c)$. This can be seen for example as follows: Since $l(s \alpha)=l\left(f_{1} \alpha\right)$, (3.9) implies that $s: S^{1} \rightarrow S^{1}$ is weakly $H_{1}$-QS in the arc metric, hence in the euclidean metric, cf. [TV, p. 113]. Since $S^{1}$ is of $\pi$-bounded turning, $s$ is $\eta$-QS with $\eta=\eta_{c}$ [TV, 2.16]. Hence $s$ is $\theta$-quasimöbius with $\theta=\theta_{c}$ by [V $\ddot{a}_{2}$, 
3.2]. Consequently, $s_{1}$ is $\theta$-quasimöbius. Since $s_{1}(\infty)=\infty, s_{1}$ is $\theta$-QS and hence (weakly) $H$-QS with $H=\theta(1)$.

Let $g: \bar{H}^{2} \rightarrow \bar{H}^{2}$ be the Beurling-Ahlfors extension of $s_{1}$. It induces a homeomorphism $f_{2}=h^{-1} g h: \bar{B}^{2} \rightarrow \bar{B}^{2}$. Then $f_{2} \mid S^{1}=s$, and $f_{2} \mid B^{2}$ is $K$-QC and $L$-bilipschitz in the hyperbolic metric of $B^{2}$ with $L=L(c), K=L^{2}$.

Step 3. The map $f=f_{1} f_{2}^{-1}: \bar{B}^{2} \rightarrow D^{*}$ is the desired map. This follows as in the proof of 3.3 from the inequalities

$$
(1-|z|) / M \leqq \delta(f(z)) \leqq M(1-|z|)
$$

where $z \in B^{2}, \quad M=M(c), \delta(w)=d(w, \partial D)$. This is proved by a rather obvious modification of the proof of the corresponding inequalities (3.6) of the half plane case. Omitting other details, we describe the construction of the arcs $C_{0}^{\prime}=f_{1} C_{0}$ and $C_{1}^{\prime}=f_{1} C_{1}$. We may assume that $7 c \delta<1-|z|$. As in the proof of (3.6), $C_{0}^{\prime}$ will be a subarc of $\partial^{*} D$ with $l\left(C_{0}^{\prime}\right)=14 c \delta$. This is possible, since $14 c \delta<2(1-|z|) \leqq 2<$ $2 \pi=l\left(\partial^{*} D\right)$. Then $C_{1}$ is chosen to be the line segment with end points $z$ and $-f^{-1}\left(w_{0}\right)$.

3.11. The quantitative version of 3.8. If $f: B^{2} \rightarrow D$ is an $L$-BLD homeomorphism, $D$ is $c$-ICA with $c=\pi L^{2}$. If $D$ is $c$-ICA and bounded with $l\left(\partial^{*} D\right)=r$, there is an $L$-BLD homeomorphism $f: B(r) \rightarrow D$ with $L=L(c)$.

\section{References}

[Ah] Ahlfors, L. V.: Lectures on quasiconformal mappings. - D. Van Nostrand Company, Inc., Princeton, New Jersey-Toronto-New York-London, 1966.

[Ge] Gehring, F. W.: Injectivity of local quasi-isometries. - Comment. Math. Helv. 57, 1982, $202-220$.

[GV] GeHRING, F. W., and J. VÄISÄLÄ: The coefficients of quasiconformality of domains in space. Acta Math. 114, 1965, 1-70.

[JK] Jerison, D. S., and C. E. Kenig: Hardy spaces, $A_{\infty}$, and singular integrals on chord-arc domains. - Math. Scand. 50, 1982, 221-247.

[Jo] John, F.: On quasi-isometric mappings, I. - Comm. Pure Appl. Math. 21, 1968, 77-110.

[La] LATfullin, T. G., (Латфуллин T. Г.): О геометрических условиях на образы прямой и окружностн при квазиизометрии плоскости. - Материалы XVIII всесоюзной научной студенческой конференщии, Новосибирск, 1980, 18-22.

[LV] Lehto, O., and K. I. VIRTANEN: Quasiconformal mappings in the plane. - Springer-Verlag, Berlin-Heidelberg-New York, 1973.

[MV] MARTIO, O., and J. VÄISÄLÄ: Elliptic equations and maps of bounded length distortion. - To appear.

[Nä] NäKKI, R.: Continuous boundary extension of quasiconformal mappings. - Ann. Acad. Sci. Fenn. Ser. A I Math. 511, 1972, 1-10.

[Po $\left.\mathrm{P}_{1}\right]$ Pommerenke, C.: Univalent functions. - Vandenhoeck \& Ruprecht, Göttingen, 1975.

[Po $\mathrm{PO}_{2}$ Pommerenke, C.: One-sided smoothness conditions and conformal mapping. - J. London Math. Soc. (2) 26, 1982, 77-82. 
[Tu $\mathrm{Tu}_{1}$ TUKIA, P.: The planar Schönflies theorem for Lipschitz maps. - Ann. Acad. Sci. Fenn. Ser. A I Math. 5, 1980, 49-72.

$\left[\mathrm{Tu}_{2}\right]$ TUKIA, P.: Extension of quasisymmetric and Lipschitz embeddings of the real line into the plane. - Ann. Acad. Sci. Fenn. Ser. A I Math. 6, 1981, 89-94.

[TV] TUKIA, P., and J. VÄIsÄLÄ: Quasisymmetric embeddings of metric spaces. - Ann. Acad. Sci. Fenn. Ser. A I Math. 5, 1980, 97-114.

[Vä̈] VÄISÄLÄ, J.: Lectures on n-dimensional quasiconformal mappings. - Lecture Notes in Mathematics 229, Springer-Verlag, Berlin-Heidelberg-New York, 1971.

[Vä] VärsäLÄ, J.: Quasimöbius maps. - J. Analyse Math. 44, 1984/85, 218-234.

University of Helsinki

Department of Mathematics

SF-00100 Helsinki

Finland

Received 9 April 1987 